OPEN ACCESS

Edited by:

Avinash Mishra,

Central Salt \& Marine Chemicals

Research Institute (CSIR), India

Reviewed by:

Anil Kumar Singh,

Indian Institute of Agricultural

Biotechnology (ICAR), India

Mohamed Shahen,

Tanta University, Egypt

${ }^{*}$ Correspondence:

Pengmin Li

lipm@nwsuaf.edu.cn

Specialty section:

This article was submitted to

Plant Abiotic Stress,

a section of the journal

Frontiers in Plant Science

Received: 10 May 2017

Accepted: 27 July 2017

Published: 09 August 2017

Citation:

Rehman RNU, You Y, Zhang L, Goudia BD, Khan AR, Li P and Ma F

(2017) High Temperature Induced Anthocyanin Inhibition and Active

Degradation in Malus profusion.

Front. Plant Sci. 8:1401.

doi: 10.3389/fpls.2017.01401

\section{High Temperature Induced Anthocyanin Inhibition and Active Degradation in Malus profusion}

\author{
Rana Naveed Ur Rehman', Yaohua You', Lei Zhang', Bachir Daoura Goudia², \\ Abdul Rehman Khan ${ }^{3}$, Pengmin $\mathrm{Li}^{1 *}$ and Fangwang $\mathrm{Ma}^{1}$ \\ 'State Key Laboratory of Crop Stress Biology for Arid Areas, College of Horticulture, Northwest A\&F University, Yangling, \\ China, ${ }^{2}$ State Key Laboratory of Crop Stress Biology for Arid Areas, College of Agronomy, Northwest A\&F University, \\ Yangling, China, ${ }^{3}$ Nuclear Institute for Agriculture and Biology, Faisalabad, Pakistan
}

The red fleshed fruits of Malus profusion represent gradual color loss during high temperature in summer, potentially due to active degradation of anthocyanin. The objective of this study was to examine both physiological and molecular evidence of anthocyanin degradation. Malus crabapple fruits were exposed to either room temperature ( $\left.R T=18 \pm 2^{\circ} \mathrm{C}: 25 \pm 2^{\circ} \mathrm{C}\right)$ or high temperature $\left(H T=33 \pm 2^{\circ} \mathrm{C}: 25 \pm 2^{\circ} \mathrm{C}\right)$ regimens (12 h: $12 \mathrm{~h}$ ) under hypoxic (2\%) or normoxic (21\%) oxygen levels. The results showed that the concentration of cyanidin 3-galactoside (cy-3-gal) was dramatically reduced following $\mathrm{HT}$ treatments due to a significant down-regulation of anthocyanin biosynthetic genes (MpCHS, MpDFR, MpLDOX, MpUFGT, and MpMYB10). Among other repressor MYBs, MpMYB15 expression was high following HT treatment of the fruit. $\mathrm{HT}$ led to the generation of a substantial concentration of $\mathrm{H}_{2} \mathrm{O}_{2}$ due to enhanced activities of superoxide dismutase (SOD), methane dicarboxylic aldehyde (MDA) content and cell sap pH value. Similarly, transcript levels of MpVHA-B1 and MpVHA-B2 were reduced which are involved in the vacuolar transportation of anthocyanin. The enzymatic degradation of anthocyanin was eventually enhanced coupled with the oxidative activities of peroxidase (POD) and $\mathrm{H}_{2} \mathrm{O}_{2}$. Conversely, the RT treatments potentially enhanced anthocyanin content by stabilizing physiological attributes (such as MDA, $\mathrm{H}_{2} \mathrm{O}_{2}$, and $\mathrm{pH}$, among others) and sustaining sufficient biosynthetic gene expression levels. Quantitative real-time PCR analysis indicated that the transcription of MpPOD1, $M P P O D 8$ and MpPOD9 genes in fruit tissues was up-regulated due to HT treatment and that hypoxic conditions seems more compatible with the responsible POD isoenzymes involved in active anthocyanin degradation. The results of the current study could be useful for understanding as well as elucidating the physiological phenomenon and molecular signaling cascade underlying active anthocyanin degradation in Malus crops.

Keywords: anthocyanin inhibition, cyanidin 3-galactoside degradation, Malus crabapple, peroxidase genes, thermal stress

\section{INTRODUCTION}

Anthocyanins are colored pigments that are ubiquitous in plants and often transitorily accumulated in the peripheral layer of mesophyll tissues (Steyn et al., 2002). Their presence is mainly pertinent to a wide range of functions at the cellular level, like the beautiful coloration of the flower/fruit parts for pollinator recruitment or seed dispersal, photo-protection, thermo-tolerance, 
and aposematic defense warnings against herbivores (Hughes et al., 2013). Moreover, such pigments are considered potential sources of nutraceuticals due to their pronounced scavenging capacity of reactive oxygen species (ROS) (Agati et al., 2012). Recently, they also have been applied extensively for anticancer, anti-inflammatory and cardiovascular protection.

Generally, fruit color reddening is considered a quality signature of enhanced antioxidant capacity and demonstrates good marketing potential; hence, fruit bagging is accredited among commercial apple cultivars to induce sensitivity to anthocyanin pigment biosynthesis (Zhang et al., 2014). The biosynthesis of anthocyanin occurs via an intricate phenylpropanoid pathway which is predominantly controlled by a plethora of structural genes [such as chalcone synthase (CHS), dihydroflavonol 4-reductase (DFR), leucoanthocyanidin oxygenase (LDOX), and UDP-glucose: flavonoid 3-O-glucosyltransferase (UFGT), among others] and a ternary complex of transcription factors (TFs) known as MYB-bHLH-WD40 (MBW) (Feng et al., 2013; Albert et al., 2014). Among the MBW complex, MYB (specifically R2R3 MYBs like $M d M Y B 1 / 10)$ is the most abundant class and is known to play a paramount role in anthocyanin pigment biosynthesis (Jiang et al., 2014). MYB TFs are induced by light and cold temperature, and their constitutive expression results in the persistent ability to bind to their own promoter in an auto-regulatory loop due to the presence of an extra five tandem repeats, resulting in increased red coloration (Espley et al., 2009; Albert et al., 2014).

Recently, R2R3 repressor MYB TFs have also been reported that inhibit anthocyanin biosynthesis, such as FaMYB1 (Fragaria sp.) (Aharoni et al., 2001), MdMYB15, MdMYB16 (Malus domestica) (Lin-Wang et al., 2011) and MtMYB2 (Medicago truncatula) (Jun et al., 2015).

Following by the biosynthesis of anthocyanin pigments, these are transported into the vacuole by the $\mathrm{H}^{+}$pumping activities of $\mathrm{H}^{+}$-ATPase (V-ATPase or VHA) and $\mathrm{H}^{+}$-pyrophosphatase (V-PPase or VHP). VHA is a multi-subunit and highly conserved proton pump that uses the energy of ATP and shows widespread expression among mature fruits (Hu et al., 2016). However, the VHP pump constitutes a single polypeptide chain, uses the phosphoanhydride bond of pyrophosphate (PPi) as its energy source, and has been extensively reported among young fruits (pear and grape) (Etienne et al., 2002, 2013; Venter et al., 2006). As fruit matures, the activities of VHP protein are greatly reduced due to the plentiful availability of ATP molecules (Etienne et al., 2013). Recently, Hu et al. (2016) demonstrated that MdVHA-B1 and MdVHA-B2 (subunits of VHA) play dominent roles in the transportation of anthocyanins and malates into vacuoles.

Previously, many reports have shown that high temperature hampers anthocyanin biosynthesis by suppressing the molecular signaling cascade and leads to a loss of biosynthetic capacity (Ubi et al., 2006; Movahed et al., 2016). However, the loss of biosynthetic capacity (only) does not confer active degradation of anthocyanin pigments. Instead, it is distinguished enzymatic and systematic process (Vaknin et al., 2005) that might differ among various crop species. Similarly, POD enzyme has been reported to be involved in the active degradation of anthocyanin in Brunfelsia calycina flowers (Zipor et al., 2015). However, comprehensive details regarding the active degradation of anthocyanin among deciduous fruits (like Malus sp.) remain largely unknown.

Malus profusion is a medium-sized tree that is grown extensively in semi-arid temperate zones of China and produces small red-fleshed fruits $(<5 \mathrm{~cm})$ that are sometimes used for traditional medicinal purposes. During summer, due to the high temperature $\left(\geq 30^{\circ} \mathrm{C}\right)$, the fruit exhibits a gradual loss of color due to concurrent turnover of anthocyanin pigments. However, molecular and physiological evidence explaining this phenomenon remain obscure. Abiotic stress like high temperature triggers the production of ROS, and hence the enzymatic antioxidant system (SOD, POD, among others) is used to protect other cellular organelles against its deleterious effects (Mittler, 2002). However, consistently elevated ROS (like $\mathrm{H}_{2} \mathrm{O}_{2}$ or $\mathrm{O}_{2}^{--}$) can damage membrane integrity and disturb many other biological processes (Ali et al., 2016).

Taken together, these findings indicate that active degradation of anthocyanin is initiated due to loss of biosynthetic capacity and the beginning of specific enzymatic degradation reactions. In the former case, loss of activity of biosynthetic enzymes/gene expression or activation of the repressor MYBs predominate, while enzymatic degradation is induced due to specific enzymes (like POD, or polyphenol oxidase). Rationally, class III POD seems to be an active candidate due to its co-localization with anthocyanin in the vacuole (Zipor et al., 2015). However, excluding a few reports such as (Movahed et al., 2016), molecular evidence of POD is still lacking due to post-translational modifications and the formation of divergent isoenzymes. In addition, Awad and de Jager (2000) found that low oxygen levels have no effect on anthocyanin biosynthesis; however, the effect of hypoxic conditions on the rate of active degradation has not been assessed before.

Fruit color is a primary determinant of the consumer's choice, but very few studies have examined gradual color loss. In the present study, we assessed both physiological and molecular evidence of the active degradation of anthocyanin among fruits of Malus profusion under hypoxic and normoxic oxygen levels. This information will be useful for understanding the mechanism of pigment degradation in Malus fruits during high temperature in summer.

\section{MATERIALS AND METHODS}

\section{Plant Materials and Treatments}

In this study, fruits were harvested from mature trees (8- to 10-years-old) of Malus profusion having a central leader growth habit with a planting distance of $3 \times 4 \mathrm{~m}$ located at Northwest A\&F University, Pomology experimental area $\left(34^{\circ} \mathrm{N}, 108^{\circ} \mathrm{E}\right)$ Yangling, Shaanxi, China. The trees were receiving standard management practices (such as irrigation, fertilization, and pruning) during 2016. Red-fleshed fruits of Malus profusion were collected at physiological maturity 110-115 days after blooming (DAB). After washing and drying at ambient 


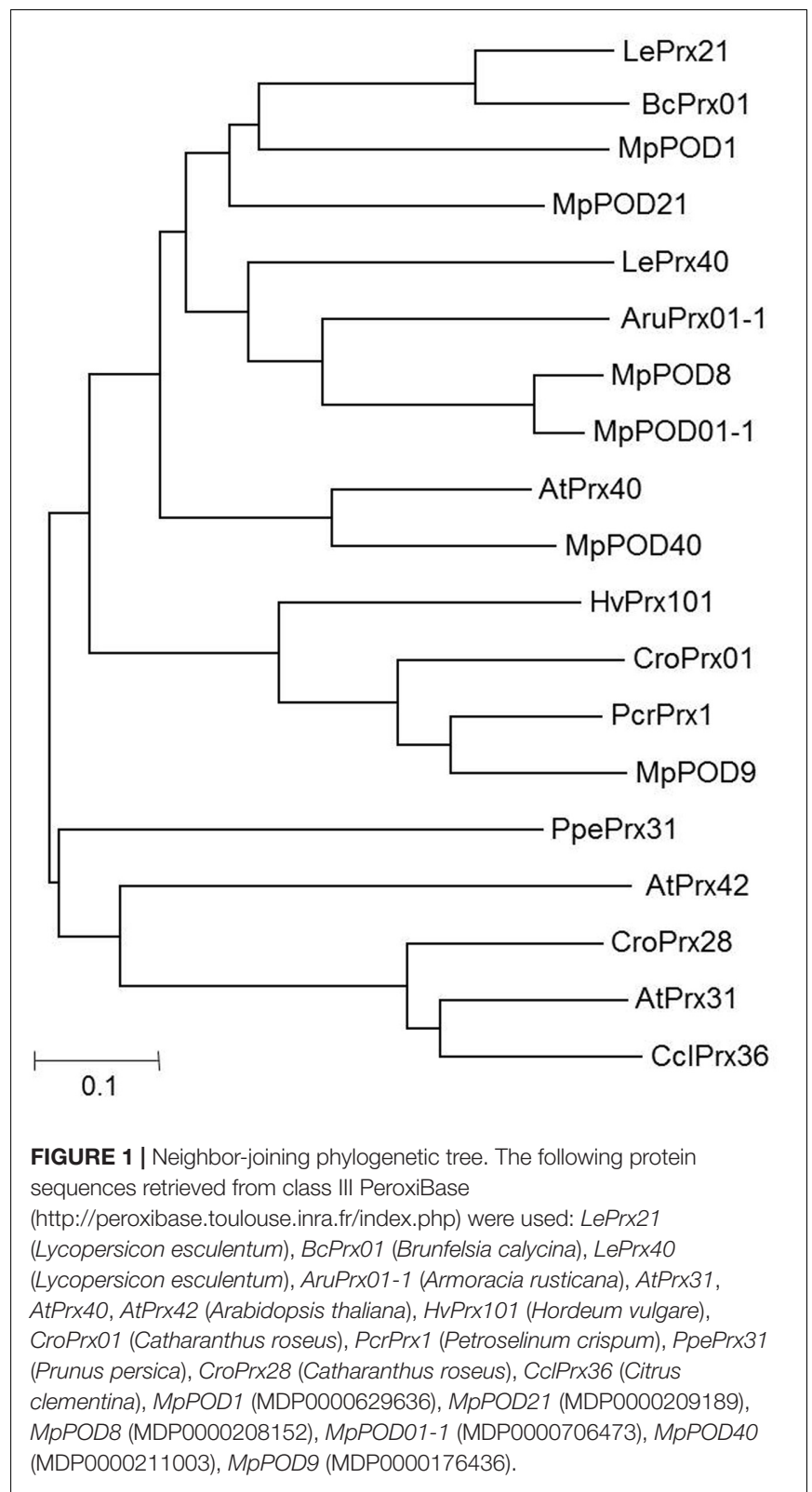

temperature $\left(25 \pm 2{ }^{\circ} \mathrm{C}\right)$, the fruits were thoroughly randomized and divided into five homogenous groups (treatments). Peel and flesh (together) of one group of fruits ( 0 day) was stored immediately at $-80^{\circ} \mathrm{C}$. While rest of treatments were exposed to two temperatures [high temperature (HT) and room temperature (RT)] with an alternative temperature regime of (12: $12 \mathrm{~h})$ under two oxygen levels viz; HT-2\% = HT $33 \pm 2{ }^{\circ} \mathrm{C}: 25 \pm 2{ }^{\circ} \mathrm{C} 2 \% \mathrm{O}_{2}$; HT- $21 \%=\mathrm{HT} 33 \pm 2{ }^{\circ} \mathrm{C}$ : $25 \pm 2{ }^{\circ} \mathrm{C}+21 \% \mathrm{O}_{2}$; RT $-2 \%=$ RT $18 \pm 2{ }^{\circ} \mathrm{C}: 25 \pm 2{ }^{\circ} \mathrm{C}+2 \%$ $\mathrm{O}_{2}$; RT $-21 \%=\mathrm{RT} 18 \pm 2{ }^{\circ} \mathrm{C}: 25 \pm 2{ }^{\circ} \mathrm{C}+21 \% \mathrm{O}_{2}$ at $85 \pm 5 \%$ $\mathrm{RH}$; for 1 week in the dark. The 2 and $21 \% \mathrm{O}_{2}$ was provided by modified packaging in plastic bags and a continuous flowthrough system, respectively, in temperature controlled chamber. After 1 week, the peel and flesh (together) were ground into a powder in liquid nitrogen using the A11 blender (IKA ${ }^{\circledR}$

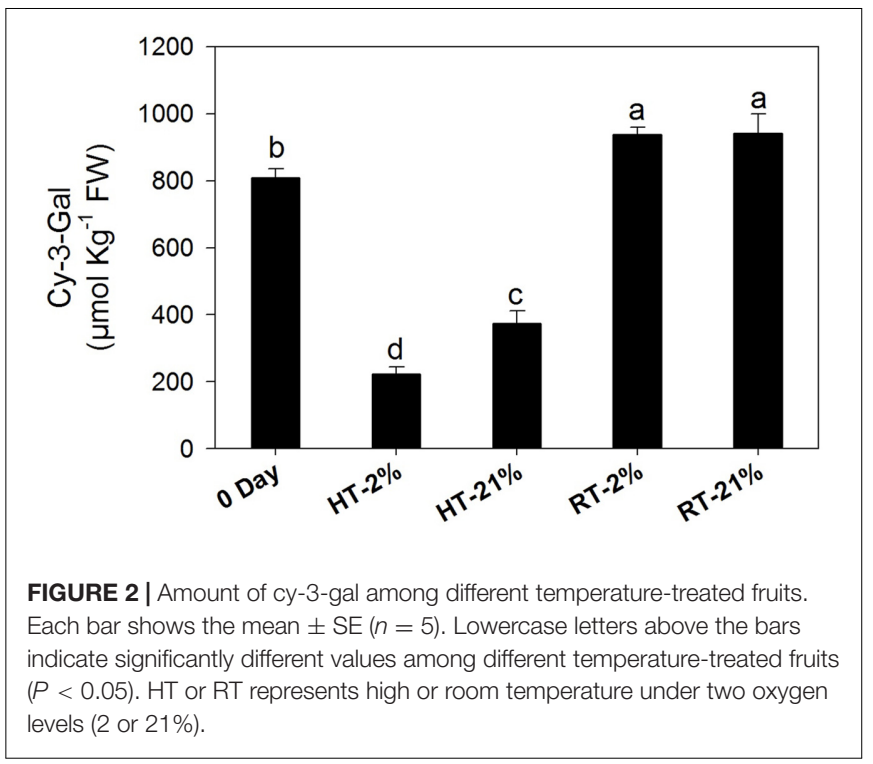

Works, Pittsburgh, PA, United States) and immediately stored at $-80^{\circ} \mathrm{C}$.

\section{The $\mathrm{pH}, \mathrm{H}_{2} \mathrm{O}_{2}$ and MDA Contents}

The $\mathrm{pH}$ values of fresh fruit were measured as reported previously (Vaknin et al., 2005). The fruit (1 g) was ground in $10 \mathrm{ml}$ deionized water using a hi-speed dispersant (XHF-D, Shanghai, China), and the $\mathrm{pH}$ was determined immediately using a Mettler Toledo $\mathrm{pH}$ meter (FE20, Shanghai, China).

The $\mathrm{H}_{2} \mathrm{O}_{2}$ concentration was assayed according to the method of Zhang et al. (2014) and expressed as $\mu \mathrm{mol} \mathrm{kg}{ }^{-1} \mathrm{FW}$ (fresh weight). Briefly, frozen samples were ground in $5 \%(\mathrm{w} / \mathrm{v})$ trichloro-acetic acid (TCA) cold extraction buffer followed by centrifugation at $13,000 \times g$ for $20 \mathrm{~min}$ at $4^{\circ} \mathrm{C}$. The supernatant was neutralized to $\mathrm{pH} 7.8$ with $\mathrm{NH}_{4} \mathrm{OH}$, and the sample was divided into two $500 \mu \mathrm{l}$ aliquots (A and B). Twenty units of catalase (CAT, EC 1.11.1.6) were added to aliquot 'A.' Both A and $\mathrm{B}$ were kept at $20^{\circ} \mathrm{C}$ for $10 \mathrm{~min}$, followed by the addition of $500 \mu \mathrm{l}$ colorimetric reagent. The colorimetric reagent was freshly prepared by mixing $0.3 \mathrm{mM}$ potassium titanium oxalate and $0.3 \mathrm{mM}$ 4-(2-pyridylazo) resorcinol monosodium salt and water at a ratio of 1:1:2 (v/v). The assay mixture was incubated at $45^{\circ} \mathrm{C}$ for $20 \mathrm{~min}$ before determining the absorbance at $508 \mathrm{~nm}$ using a UV-vis spectrophotometer (2450 Shimadzu, Tokyo, Japan).

The MDA content was determined as previously described (Xue et al., 2015) after minor modifications and expressed as the $\mu \mathrm{mol} \mathrm{g}{ }^{-1} \mathrm{FW}$. Frozen samples were ground in buffer containing ethanol: water $(80: 20 \mathrm{v} / \mathrm{v})$, and centrifuged at 5,000 $\times g$ for $10 \mathrm{~min}$ at $4^{\circ} \mathrm{C}$. The supernatant $(1 \mathrm{ml})$ was mixed vigorously with $0.65 \%$ thiobarbituric acid (in $10 \%$ TCA), heated at $95^{\circ} \mathrm{C}$ until boiling and promptly cooled on ice and centrifuged at $3,500 \times g$ for $2 \mathrm{~min}$ to remove the flocculate. The clear supernatant was used to determine the absorbance at 450, 532, and $600 \mathrm{~nm}$ using a UV-vis spectrophotometer (2450 Shimadzu, Tokyo, Japan). 


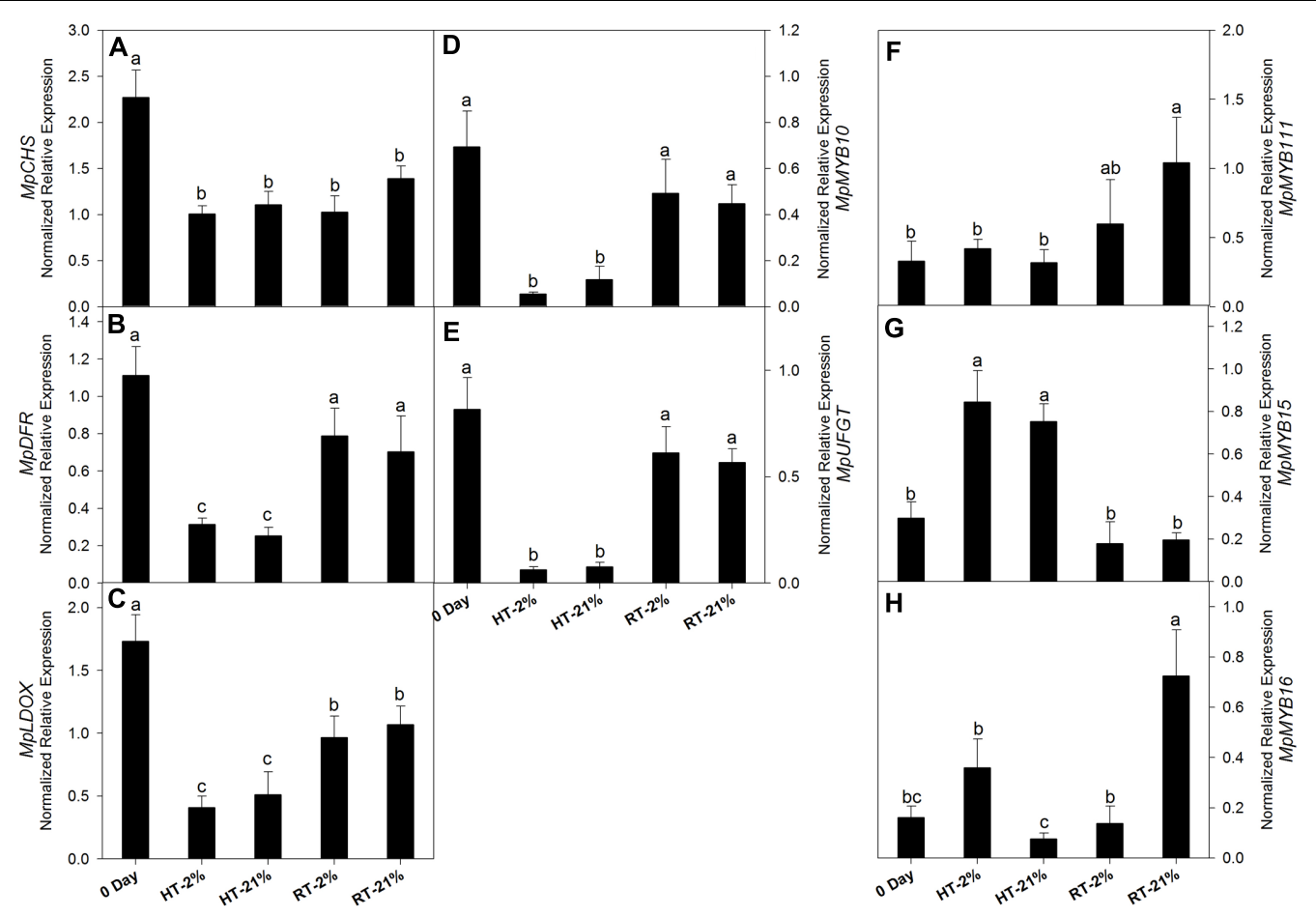

FIGURE 3 | Relative transcription levels of biosynthetic genes and repressor MYBs. MpCHS (A), MpDFR (B), MpLDOX (C), MpMYB10 (D), MpUFGT (E) and repressor MYBs MpMYB111 (F), MpMYB15 (G) and MpMYB16 (H) among different temperature-treated fruits. Each bar is the mean \pm SE $(n=3)$. Lowercase letters above the bars indicate significantly different values among different temperature treated fruits $(P<0.05)$. HT or RT represents high or room temperature under two oxygen levels (2 or $21 \%)$.

\section{Anthocyanin Quantification}

Anthocyanin was quantified by high-performance liquid chromatograph (HPLC) as described previously (Zhang et al,, 2014). Briefly, frozen fruit tissues were ground using phenolic extract solution containing methanol: formic acid (70\%: $2 \%$ ), centrifuged at $12,500 \times g$ for $20 \mathrm{~min}$ at $4^{\circ} \mathrm{C}$ and the supernatant was filtered using a syringe filter $(0.45 \mu \mathrm{m})$ prior to HPLC injection (HP1200 Shimadzu, Tokyo, Japan). The HP1200 was equipped with a diode array detector (Agilent technologies, Palo Alto, CA, United States), an Inertsil ODS-3 Guard Column $(4.0 \mathrm{~mm} \times 10 \mathrm{~mm} ; 5.0 \mu \mathrm{m}$ particle size; GL Sciences, Tokyo, Japan), and an Inertsil ODS-3 column $(4.6 \mathrm{~mm} \times 250 \mathrm{~mm}$; $5.0 \mu \mathrm{m}$ particle size; GL Sciences, Tokyo, Japan). Solvent A and B contained $10 \%$ formic acid in water and $10 \%$ formic acid in HPLC-grade acetonitrile, respectively. The order of the gradient used was as follows: $95 \%$ A (0 $\mathrm{min}), 85 \%$ A ( $25 \mathrm{~min}$ ), $78 \%$ A (42 $\mathrm{min}), 64 \% \mathrm{~A}(60 \mathrm{~min})$, and $95 \% \mathrm{~A}(65 \mathrm{~min})$. The flow rate was $1.0 \mathrm{ml} \mathrm{min}{ }^{-1}$ at $30^{\circ} \mathrm{C}$, the post-run time was $10 \mathrm{~min}$, and monitoring was performed at $520 \mathrm{~nm}$ for cy-3-gal. The cy-3-gal peak was identified by comparing retention times and UV spectra with reliable standards. The concentration was determined based on the peak area and calibration curves derived from standards of the corresponding compound. All phenolic standards were obtained from Sigma-Aldrich (St. Louis, MO, United States), ExtraSynthese (Genay, France), and AApin Chemicals (Abingdon, Oxon, United Kingdom).

\section{Enzyme Assays}

Frozen samples of Malus profusion were ground in extraction buffer containing $0.014 \mathrm{M} \quad \beta$-mercaptoethanol, $0.002 \mathrm{M}$ EDTA-Na $2,0.5 \%$ Triton X-100, $1 \%$ BSA, 0.005 M DDT, $10 \%$ glycerol and $0.1 \mathrm{M}$ Tris- $\mathrm{HCl}(\mathrm{pH} 7.5)$. After thorough homogenization, the samples were centrifuged at $12,000 \times g$ for $15 \mathrm{~min}$ at $4^{\circ} \mathrm{C}$. The supernatant was quickly used for further analysis.

The activity of peroxidase (POD; EC 1.11.1.7) was determined by guaiacol oxidation according to Ali et al. (2016) with slight modifications and expressed as $\mu \mathrm{mol} \mathrm{m}^{-2} \mathrm{~s}^{-1}$. The $1 \mathrm{ml}$ reaction mixture contained $0.05 \mathrm{M}$ sodium phosphate buffer ( $\mathrm{pH} 7.0$ ), $0.01 \mathrm{M} \mathrm{H}_{2} \mathrm{O}_{2}, 0.01 \mathrm{M}$ guaiacol and $10 \mu \mathrm{l}$ of the enzyme extract. The reaction was initiated by adding $\mathrm{H}_{2} \mathrm{O}_{2}$, and the activity was determined by monitoring the change in absorbance at $470 \mathrm{~nm}$ using a UV-Vis spectrophotometer (2450 Shimadzu, Tokyo, Japan).

The activity of superoxide dismutase (SOD; EC 1.15.1.1) was determined by monitoring the inhibition of the photochemical reduction of nitroblue tetrazolium (NBT) according to previously described methods (Štajner and Popović, 2009) and expressed as $10^{5} \mathrm{U} \mathrm{kg}^{-1} \mathrm{FW}$. The $1 \mathrm{ml}$ reaction mixture contained $580 \mu \mathrm{l}$ of Tris- $\mathrm{HCl} \mathrm{pH} 7.5(0.1 \mathrm{M}), 100 \mu \mathrm{l}$ of methionine $(0.065 \mathrm{M}), 100 \mu \mathrm{l}$ of NBT $(50 \mu \mathrm{M}), 200 \mu \mathrm{l}$ of riboflavin $(20 \mu \mathrm{M})$, and $20 \mu \mathrm{l}$ of the enzyme extract. The test tubes wrapped in aluminum foil were vigorously mixed and irradiated for $30 \mathrm{~min}$ 


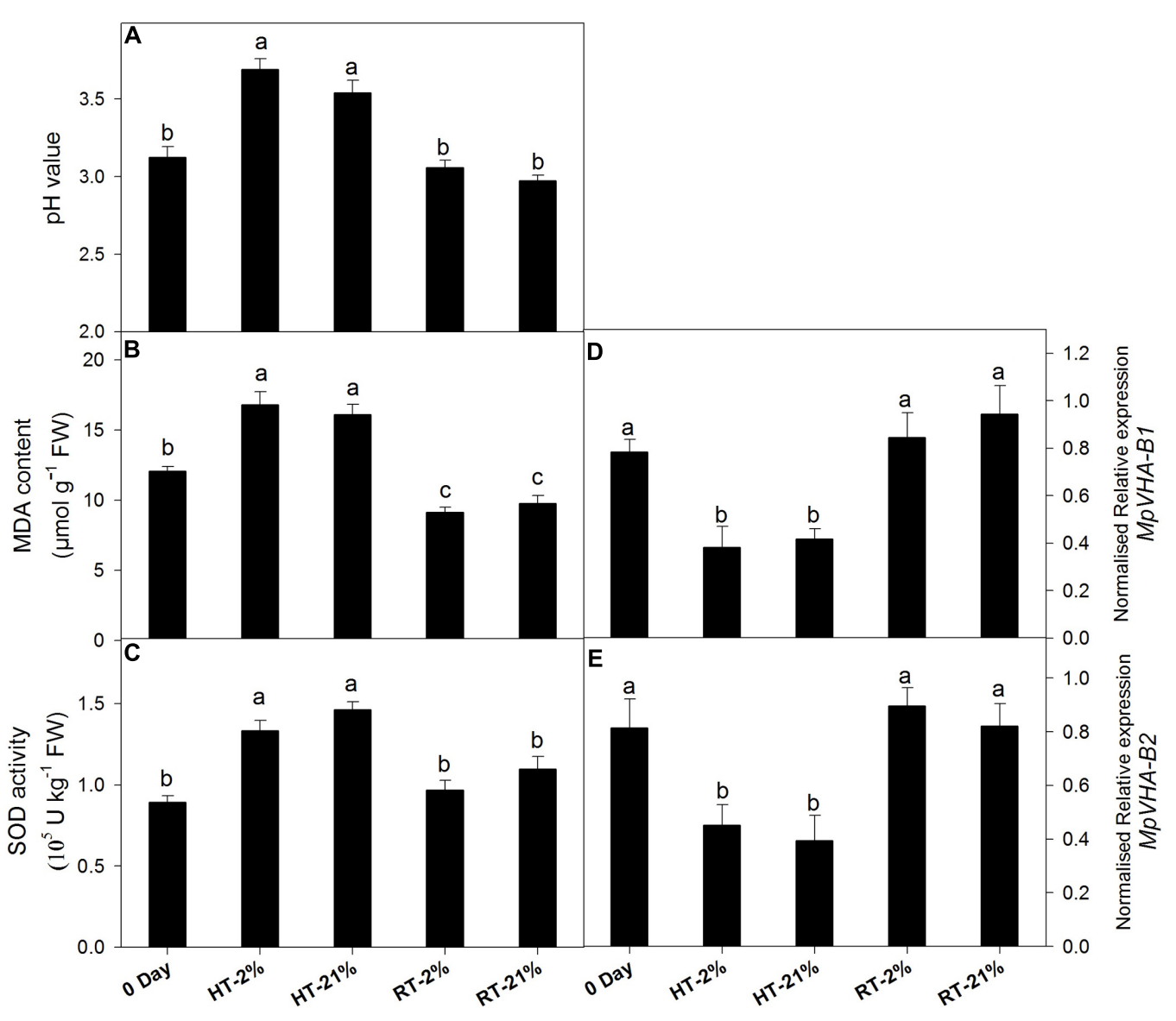

FIGURE 4 | Effect of hypoxic thermal stress on other physiological events. The pH value (A), MDA content (B), SOD activity (C) and relative transcript levels of MpVHA-B1 (D) and MpVHA-B2 (E) among different temperature-treated fruits. For $(\mathbf{A}-\mathbf{C})$ and $(\mathbf{D}, \mathbf{E})$ each bar is the mean $\pm \mathrm{SE}(n=5)$ and $(n=3)$, respectively. Lowercase letters above the bars indicate significantly different values among different temperature-treated fruits $(P<0.05)$. HT or RT represents high or room temperature under two oxygen levels (2 or $21 \%)$.

under fluorescent light before determining the absorbance at $560 \mathrm{~nm}$ using a UV-Vis spectrophotometer (2450 Shimadzu, Tokyo, Japan).

\section{Quantitative Real-time PCR Analyses}

Fruit RNA was extracted using the SDS-phenol method according to Zhang et al. (2014) and quantitated using a NanoDrop 2000 micro-volume spectrophotometer (Thermo Fisher Scientific, Waltham, MA, United States) at 260 and $280 \mathrm{~nm}$. RNA quality and integrity were assessed by $1.2 \%$ agarose gel electrophoresis, and the gel was stained with ethidium bromide. First-strand cDNA was synthesized from $1 \mu \mathrm{g}$ of total RNA using the reverse PrimeScript RT reagent Kit ${ }^{\mathrm{TM}}$ (Takara, Dalian, China) according to the manufacturer's instructions. cDNA amplifications were carried out with primers for MpCHS, MpDFR, MpLDOX, MpUFGT, MpMYB10, MpMYB15, MpMYB16 MpMYB111, MpPOD1, MpPOD21, MpPOD40, MpPOD01-1, MpPOD8, MpPOD9, MpVHA-B1, and MpVHA-B2 (Supplementary Table S1). All reactions were performed with three biological replicates containing $10 \mu \mathrm{l}$ of SYBR Green MasterMix (SYBR Premix EX Taq ${ }^{\mathrm{TM}}$, Dalian, China), $2 \mu \mathrm{l}$ of cDNA, $6.4 \mu \mathrm{l}$ of nuclease-free water, and $0.8 \mu \mathrm{l}$ of each primer to a final volume of $20 \mu \mathrm{l}$. The Q5 Multicolor Real-Time PCR Detection System (Bio-Rad Laboratories, Hercules, CA, United States) was used for gene quantification. MpActin was used to standardize the cDNA samples for different genes (Kumar and Singh, 2015), and hence the results are presented as the normalized relative expression. The relative expression levels of target genes were calculated according to the $2^{-\Delta \Delta C_{\mathrm{T}}}$ method (Zhang et al., 2014).

\section{Gene Selection and Phylogenetic Analyses}

The nucleotide sequences of $M p C H S, M p D F R, M p L D O X$, MpUFGT, MpMYB10, MpMYB15, MpMYB16 MpMYB111, $M p V H A-B 1$, and $M p V H A-B 2$ were obtained from the Malus genome database ${ }^{1}$. Similarly, the protein sequences of class III POD among Malus sp. (MpPOD21, MpPOD40, $M p P O D 01-1, M p P O D 8$, and $M p P O D 9)$ were retrieved from

\footnotetext{
${ }^{1}$ https://www.rosaceae.org/
} 
PeroxiBase $^{2}$. A BLAST analysis of the protein sequences was performed using the Malus genome databases ${ }^{3}$, and nucleotide sequences were obtained. For the nucleotide sequence of $M P P O D 1$, the closest homologs of the protein sequences of $B c P r x 01$ were selected from the PeroxiBase database and applied for a BLAST search against the Malus genome database. For phylogenetic tree construction of POD genes, the MUSCLE program was used with default parameters (Figure 1).

\section{Statistical Analysis}

All data were subjected to analysis of variance with SPSS 16.0 for Windows (SPSS Inc., Chicago, IL, United States). Data were analyzed using one-way analysis of variance (ANOVA) followed by the least significant difference (LSD) computed at $P<0.05$. Five biological replicates for all physiological parameters $(n=5)$ and three for qPCR $(n=3)$ were used for all statistical analysis.

\section{RESULTS}

\section{High Temperature Reduced Anthocyanin Content}

Cy-3-gal was identified as main anthocyanin pigment by HPLC analysis. The concentration of cy-3-gal was markedly $(P<0.05)$ diminished under hypoxic (67\%) and normoxic (54\%) high-temperature treatments, while RT treatments showed significant biosynthesis of cy-3-gal regardless of the oxygen level (Figure 2).

\section{High Temperature Induced Down-regulation of Biosynthetic Genes and Epistatic Gene Interactions with Repressor MYBs}

Cy-3-gal is a product of the phenylpropanoid pathway. To evaluate the biosynthetic potential among different treatments, the transcriptional level of key genes and repressor MYBs were assessed (Figures 3A-H). In general, relative gene expressions for all the putative biosynthetic genes were tremendously high on day 0 . After fruit detachment from the tree, HT $\left(2 / 21 \% \quad \mathrm{O}_{2}\right.$ on average) treatments exhibited a significant $(P<0.05)$ down-regulation of $M p C H S$ (twofold), MpDFR (fourfold), MPLDOX (3.8-fold), MPUFGT (12-fold) and MpMYB10 (ninefold) (Figures 3A-E). However, the expression levels were maintained by $50-70 \%$ (on average) for the RT treatments.

In the case of repressor MYBs like MpMYB111, MpMYB15 and MpMYB16 (Figures 3F-H), not all of them correlated with the loss of biosynthetic capacity. However, MpMYB15 showed an integrative higher expression level $(P<0.05)$ at HT under hypoxic (2.8-fold) and normoxic (2.5-fold) conditions (Figure 3G).

${ }^{2} \mathrm{http}: / /$ peroxibase.toulouse.inra.fr/index.php

${ }^{3} \mathrm{https} / / /$ www.rosaceae.org/

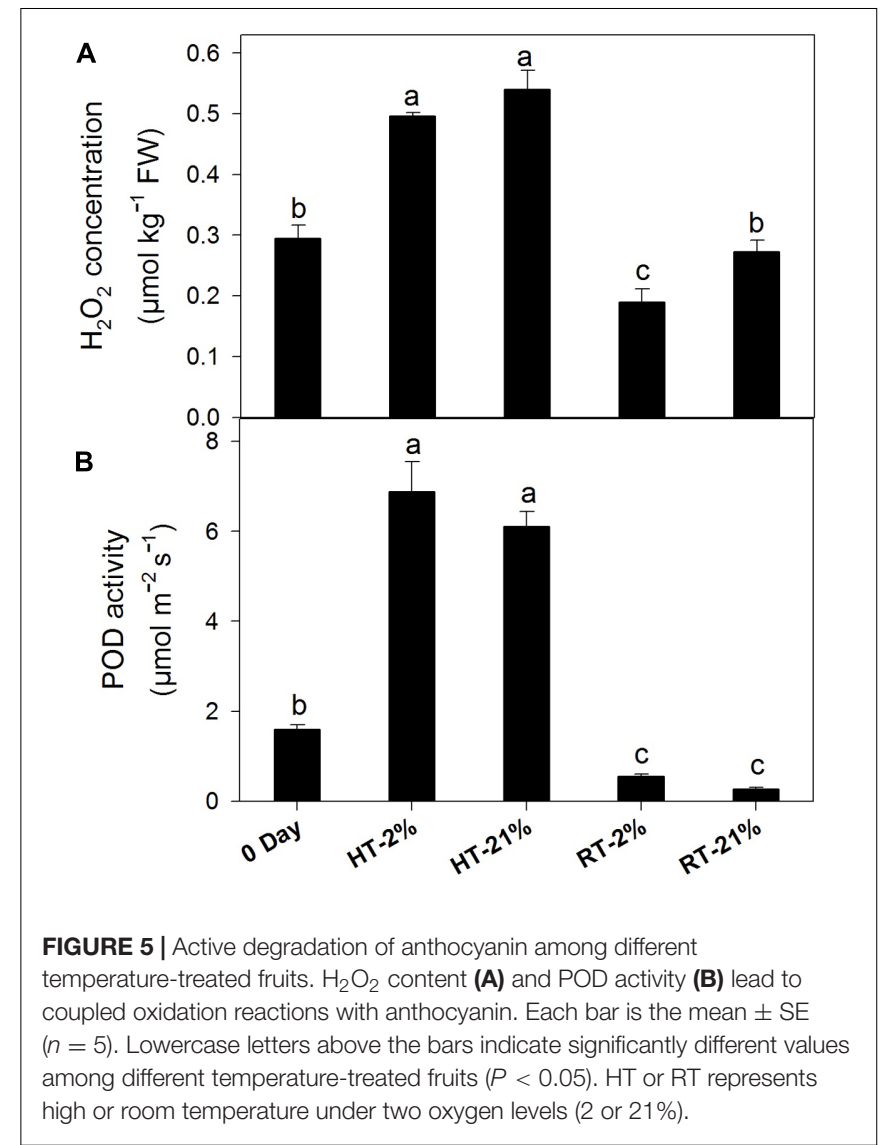

\section{Role of Other Physiological and Molecular Attributes at High Temperature}

A loss of anthocyanin biosynthetic potential and inhibition by repressor MYBs was observed for the HT treatments. To assess the physiological phenomenon regarding remaining the cy-3-gal concentration in the vacuole, we quantified the MDA content, SOD activity, $\mathrm{pH}$ value, and transcription levels of the sub units of vacuolar pump ( $M p V H A-B 1$ and $M p V H A-B 2$ ) (Figures 4A-E).

High temperature treatment showed an augmented MDA content (1.5-fold) and $\mathrm{pH}$ values (1.15-fold) (Figures $4 \mathbf{4 A}, \mathbf{B}$ ). Transcript levels of MpVHA-B1 and MpVHA-B2 were also significantly $(P<0.05)$ lower for HT treatments compared with 0 day or RT treatments (Figures $4 \mathrm{D}, \mathrm{E}$ ). However, the RT treatments showed significantly $(P<0.05)$ reduced MDA contents and inconsistent variations in $\mathrm{pH}$ values. The activity of SOD was significantly $(P<0.05)$ increased for HT treatment (Figure 4C).

\section{Active Enzymatic Degradation of Anthocyanin}

Abiotic stress triggers $\mathrm{H}_{2} \mathrm{O}_{2}$ production, which together with POD can undergo coupled oxidation with anthocyanin. $\mathrm{H}_{2} \mathrm{O}_{2}$ was higher for heated fruits and significantly reduced $(P<0.05)$ for hypoxic RT (Figure 5A). Similarly, HT treatments showed 


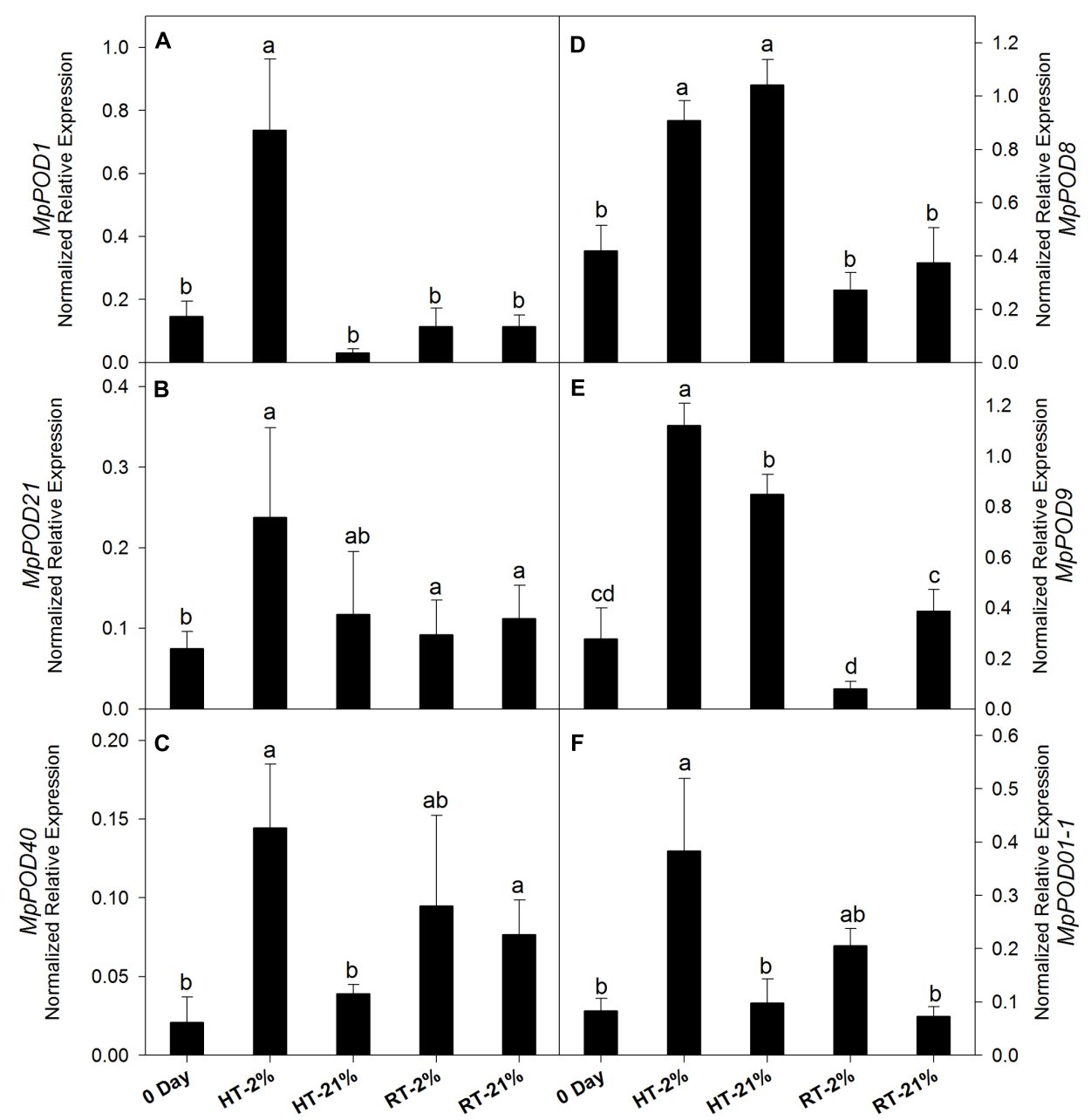

FIGURE 6 | Relative expression levels of POD genes among different temperature-treated fruits. Transcription level of MpPOD1 (A), MpPOD21 (B), MpPOD40 (C), MPPOD8 (D), MpPOD9 (E) and MpPOD01-1 (F) genes. Each bar is the mean $\pm \mathrm{SE}(n=3)$. Lowercase letters above the bars indicate significantly different values among different temperature-treated fruits $(P<0.05)$ calculated using one-way analysis of variance (ANOVA) followed by the least significant difference (LSD). HT or RT represents high or room temperature under two oxygen levels (2 or $21 \%)$.

elevated $(P<0.05)$ enzymatic activity regardless of the oxygen level (average of fourfold), while it remained at less than half of the original level for RT treatments (Figure 5B).

The expression levels of different POD genes are presented in (Figures 6A-F). MPPOD8 and MPPOD9 showed higher $(P<0.05)$ expression in response to HT treatment (Figures 6D,E). In contrast, $M p P O D 1, M p P O D 21, M p P O D 40$, and $M p P O D 01-1$ exhibited relatively high transcript levels for only hypoxic HT (Figures 6A-C,F). However, transcription levels remained non-consistent for RT treatments except MPPOD9 which shows significant $(P<0.05)$ variation at different oxygen levels (Figure 6E).

\section{DISCUSSION}

Immense literature is available for anthocyanin biosynthesis, but very few studies have assessed active degradation among Malus species. In the current study, we have provided physiological and genetic evidences of color loss in Malus profusion fruit.

Concentration of anthocyanin was highly decreased following HT treatment due to the thermal sensitivity of MpMYB10 and late biosynthetic genes (MpDFR, MpANS, and MpUFGT), as previously reported (Steyn et al., 2004; Ubi et al., 2006). Regarding the RT treatments, anthocyanin accumulation was significantly enhanced, while the transcript levels of biosynthetic genes were not significant at 0 day (Figures 3B,D,E). This phenomenon might be due to improved pigment stability at room temperature, since in orchards, fruits are exposed to abbreviated heat stress (nearly $4 \mathrm{~h}$ ) at noon and a net anthocyanin at day 0 counterbalance by simultaneous biosynthesis and active degradation. However, after RT exposure, the rate of anthocyanin accumulation was significantly enhanced, indicating that the stability of anthocyanin plays an inevitable role in the overall pigment content (Shaked-Sachray et al., 2002; Sinilal et al., 2011). 


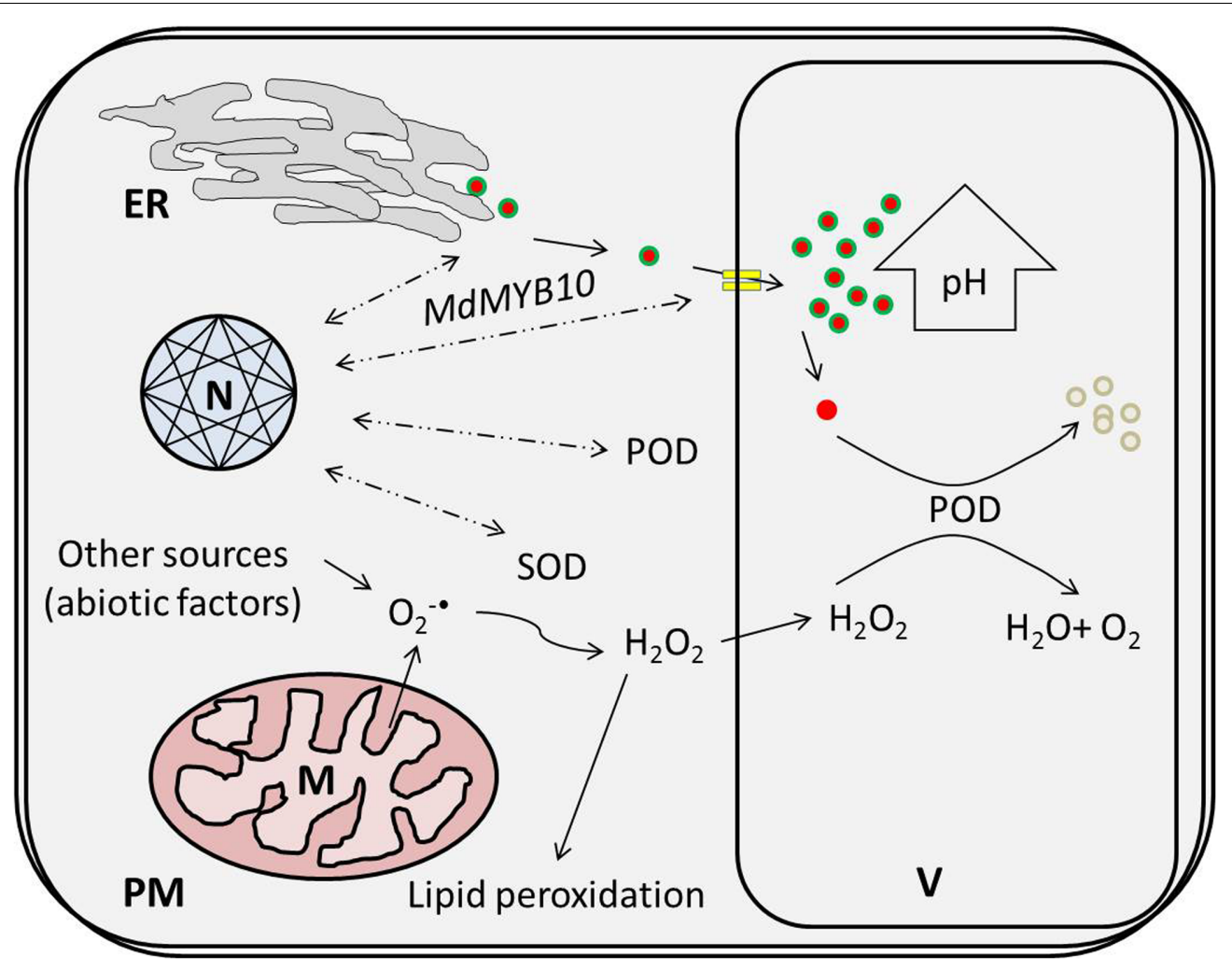

FIGURE 7 | High temperature-induced physiological changes at the cellular level lead to the active degradation of cy-3-gal. ROS produced via thermal induction leads to a coupled oxidative reaction with POD and phenolic in the vacuole. ER, N, V, M, PM, POD, and SOD represent the endoplasmic reticulum, nucleus, vacuole, plasma membrane, peroxidase, and superoxide dismutase, respectively.

Our results corroborated the findings of Lin-Wang et al. (2011) showing that MPMYB15 functioned well in fruit at a high temperature (Figure 3G). We also speculate that MpMYB111/MpMYB16 (Figures 3F,H) have higher expression levels for RT treatments (unheated), while the concentrations of anthocyanin are not as markedly decreased. Although the interplay between biosynthetic or repressor MYBs has not been completely understood, these results suggest that biosynthetic MYB play a dominant role over repressor MYB under favorable conditions, which might be due to epistatic interactions between the MBW complex and repressor MYB, relating to functional congruency or redundancy and resulting in phenotypic plasticity (Lightbourn et al., 2007; Nakatsuka et al., 2013).

Regarding physiological features, the concentration of $\mathrm{H}_{2} \mathrm{O}_{2}$ increased in response to the HT treatments, which might have been due to mitochondria, which represent a predominant site of ROS production among non-photosynthetic tissues in the dark (Navrot et al., 2007). In mitochondria, dysfunctional cytochrome c oxidase, as an essential component of the electron transfer chain (ETC), has been associated with superoxide $\left(\mathrm{O}_{2}^{\bullet}-\right.$ ) free radical generation (due to attack of excessive ROS) and is considered to confer extreme deleterious effects on other biological activities (Sedlák et al., 2010; Shafi et al., 2015). Enhanced SOD activity in response to $\mathrm{HT}$ treatments supports the hypothesis that $\mathrm{O}_{2}^{\bullet-}$ are promptly scavenged to $\mathrm{H}_{2} \mathrm{O}_{2}$ (being less deleterious)
(Navrot et al., 2007) and that this consistent increment in $\mathrm{H}_{2} \mathrm{O}_{2}$ concentration leads to membrane oxidation like lipid peroxidation, disruption of enzymatic inhibition and DNA/RNA damage (Figure 7) (Mittler, 2002). Hence, the MDA content was improved nearly 1.5 -fold for the HT treatments in comparison to 0 day.

Moreover, the expression levels of MPVHA-B1 and $M p V H A-B 2$ decreased in response to HT treatment, suggesting that vacuolar transportation of anthocyanin is dramatically reduced. Interestingly, three isoforms of VHA protein are present in apple, which are encoded by three genes (MpVHA-B1, $M p V H A-B 2$, and MpVHA-B3) containing MYB recognition sites in their promoter regions. By using EMSA, ChIP-PCR and GUS assays, Hu et al. (2016) demonstrated that MdMYB1/10 only binds to MdVHA-B1 and MdVHA-B2 (not MdVHA-B3) to facilitate the coupled transportation of malate and anthocyanin into the vacuole. However, reduced expression levels of MpVHA-B1 and MpVHA-B2 are a hallmark of perturbed transportation of anthocyanin into the vacuole.

In addition, PODs are multifunctional enzymes, and their thermal induction has been well proposed to provide protection against $\mathrm{H}_{2} \mathrm{O}_{2}$ during stress conditions in apple (Requesens et al., 2014). Similarly in strawberry fruits, enhanced POD activity is supposed to be associated with membrane damage recovery against gradual heat stress (Gulen and Eris, 2004). 
Higher plants typically contain 8-15 kinds of POD families and are generally classified based on their isoelectric points (acidic or basic) isoenzymes (Pearse et al., 2005). Among those families, the role of class III POD (basic) has been well demonstrated and characterized during the active degradation of anthocyanin (Vaknin et al., 2005; Zhang et al., 2005; Zipor et al., 2015). However, it is difficult to obtain precise molecular evidence about class III POD genes involved in the active degradation of anthocyanin due to multigene family control and the formation of divergent isoenzymes during post-transcriptional modifications (Movahed et al., 2016). In the present study, hypoxic high temperature induced PODs (MpPOD01-1, MpPOD9, and MpPOD1) have been elucidated. Their induction might be regulated by promoter sensitivity of several abiotic stresses. The promoters of different abiotic stress-responsive genes have varying number of regulatory elements that enhance the expression profile of certain genes, such as LTRE (low temperature-responsive element) or ABRE (abscisic acid-responsive element) are the key stimulant for regulating stress inducible gene expressions (Bhuria et al., 2016). These abiotic stresses (thermal, dehydration or others) independently or together co-regulate the promoters of stress responsive genes. Induction of specific POD genes might be due to the coupled effect of hypoxic conditions and thermal stress since ultra-low oxygen storage at cold temperature have negligible effects on the biosynthesis of anthocyanin and other phenolic compounds (Awad and de Jager, 2000). Another possible reason for the low anthocyanin content in hypoxic thermal conditions may be attributed to the disturbed respiratory quotient, resulting in the production of excessive acetaldehyde (or other similar compounds) during anaerobic respiration, which sequentially affects other metabolic reactions (Thewes et al., 2017).

Finally, thermal stress reduces the biosynthetic potential of anthocyanin and triggers ROS generation. It also induces specific POD genes due to the coupled effect of HT and low oxygen. Enhanced POD and $\mathrm{H}_{2} \mathrm{O}_{2}$ activities induce sequential coupled oxidation of anthocyanin pigment and cause color loss during high temperatures in Malus crabapple fruits, as illustrated in Figure 7 .

\section{REFERENCES}

Agati, G., Azzarello, E., Pollastri, S., and Tattini, M. (2012). Flavonoids as antioxidants in plants: location and functional significance. Plant Sci. 196, 67-76. doi: 10.1016/j.plantsci.2012.07.014

Aharoni, A., De Vos, C. H. R., Wein, M., Sun, Z., Greco, R., Kroon, A., et al. (2001). The strawberry FaMYB1 transcription factor suppresses anthocyanin and flavonol accumulation in transgenic tobacco. Plant J. 28, 319-332. doi: 10.1046/j.1365-313X.2001.01154.x

Albert, N. W., Davies, K. M., Lewis, D. H., Zhang, H., Montefiori, M., Brendolise, C., et al. (2014). A conserved network of transcriptional activators and repressors regulates anthocyanin pigmentation in eudicots. Plant Cell 26, 962-980. doi: 10.1105/tpc.113.122069

Ali, S., Sattar, A., Ullah, A., and Shahid, M. (2016). Effect of controlled atmosphere storage on pericarp browning, bioactive compounds and antioxidant enzymes of litchi fruits. Food Chem. 206, 18-29. doi: 10.1016/j.foodchem.2016.03.021

Awad, M. A., and de Jager, A. (2000). Flavonoid and chlorogenic acid concentrations in skin of 'Jonagold' and 'Elstar' apples during and after regular

\section{CONCLUSION}

Anthocyanin biosynthesis is inhibited and degraded at high temperatures due to up-regulation of the repressor MpMYB15 and coupled oxidation reactions of POD and $\mathrm{H}_{2} \mathrm{O}_{2}$, respectively. Vacuolar transportation of anthocyanin pigments was also reduced during thermal stress due to down-regulation of $M p V H A-B 1$ and $M p V H A-B 2$. A lack of information about the MBW complex and repressor $M Y B$ interplay is a major handicap in distinguishing the loss of anthocyanin biosynthetic capacity and its suppression. However, RT treatments with modified packaging ( 2 and $21 \% \mathrm{O}_{2}$ ) sustained better physiological attributes over the same duration. Further studies should assess the functional characterization of POD genes and their divergent isoenzymes. Such information will be useful for understanding physiological and molecular signaling cascades in active anthocyanin degradation, thus improving the fruit quality and economic traits.

\section{AUTHOR CONTRIBUTIONS}

All authors made contribution to the experiment work and manuscript write up. RNUR made substantial contribution to the design of the work, acquisition, analysis, and interpretation of data and drafting the manuscript. YY and LZ analyzed data. BDG and ARK revised manuscript. PL and FM made contribution to the design of the work, interpretation of data and critically revision of the manuscript. All authors made contribution to the approval of the final version of the manuscript to be published and agreed to be accountable for all aspects of the work in ensuring that questions related to the accuracy or integrity of any part of the work are appropriately investigated and resolved.

\section{SUPPLEMENTARY MATERIAL}

The Supplementary Material for this article can be found online at: http://journal.frontiersin.org/article/10.3389/fpls.2017.01401/ full\#supplementary-material

and ultra low oxygen storage. Postharvest Biol. Technol. 20, 15-24. doi: 10.1016/ S0925-5214(00)00116-2

Bhuria, M., Goel, P., Kumar, S., and Singh, A. K. (2016). The promoter of AtUSP is co-regulated by phytohormones and abiotic stresses in Arabidopsis thaliana. Front. Plant Sci. 7:1957. doi: 10.3389/fpls.2016. 01957

Espley, R. V., Brendolise, C., Chagne, D., Kutty-Amma, S., Green, S., Volz, R., et al. (2009). Multiple repeats of a promoter segment causes transcription factor autoregulation in red apples. Plant Cell 21, 168-183. doi: 10.1105/tpc. 108.059329

Etienne, A., Génard, M., Lobit, P., and Bugaud, C. (2013). What controls fleshy fruit acidity? A review of malate and citrate accumulation in fruit cells. J. Exp. Bot. 64, 1451-1469. doi: 10.1093/jxb/ert035

Etienne, C., Moing, A., Dirlewanger, E., Raymond, P., and Rothan, C. (2002). Isolation and characterization of six peach cDNAs encoding key proteins in organic acid metabolism and solute accumulation: involvement in regulating peach fruit acidity. Physiol. Plant. 37, 259-270. doi: 10.1034/j.1399-3054.2002. 1140212.x 
Feng, F., Li, M., Ma, F., and Cheng, L. (2013). Phenylpropanoid metabolites and expression of key genes involved in anthocyanin biosynthesis in the shaded peel of apple fruit in response to sun exposure. Plant Physiol. Biochem. 69, 54-61. doi: 10.1016/j.plaphy.2013.04.020

Gulen, H., and Eris, A. (2004). Effect of heat stress on peroxidase activity and total protein content in strawberry plants. Plant Sci. 166, 739-744. doi: 10.1016/j. plantsci.2003.11.014

Hu, D., Sun, C., Ma, Q., You, C., Cheng, L., and Hao, Y. (2016). MdMYB1 regulates anthocyanin and malate accumulation by directly facilitating their transport into vacuoles in apples. Plant Physiol. 170, 1315-1330. doi: 10.1104/pp.15.01333

Hughes, N. M., Carpenter, K. L., and Cannon, J. G. (2013). Estimating contribution of anthocyanin pigments to osmotic adjustment during winter leaf reddening. J. Plant Physiol. 170, 230-233. doi: 10.1016/j.jplph.2012.09.006

Jiang, R., Tian, J., Song, T., Zhang, J., and Yao, Y. (2014). The Malus crabapple transcription factor McMYB10 regulates anthocyanin biosynthesis during petal coloration. Sci. Hortic. 166, 42-49. doi: 10.1016/j.scienta.2013.12.002

Jun, J. H., Liu, C., Xiao, X., and Dixon, R. A. (2015). The transcriptional repressor MYB2 regulates both spatial and temporal patterns of proanthocyandin and anthocyanin pigmentation in Medicago truncatula. Plant Cell 27, 2860-2879. doi: 10.1105/tpc.15.00476

Kumar, G., and Singh, A. K. (2015). Reference gene validation for qRT-PCR based gene expression studies in different developmental stages and under biotic stress in apple. Sci. Hortic. 197, 597-606. doi: 10.1016/j.scienta.2015.10.025

Lightbourn, G. J., Stommel, J. R., and Griesbach, R. J. (2007). Epistatic interactions influencing anthocyanin gene expression in Capsicum annuит. J. Am. Soc. Hort. Sci. 132, 824-829.

Lin-Wang, K., Micheletti, D., Palmer, J., Volz, R., Lozano, L., Espley, R., et al. (2011). High temperature reduces apple fruit colour via modulation of the anthocyanin regulatory complex. Plant Cell Environ. 34, 1176-1190. doi: $10.1111 / j .1365-3040.2011 .02316 . x$

Mittler, R. (2002). Oxidative stress, antioxidants and stress tolerance. Trends Plant Sci. 7, 405-410. doi: 10.1016/S1360-1385(02)02312-9

Movahed, N., Pastore, C., Cellini, A., Allegro, G., Valentini, G., and Zenoni, S. (2016). The grapevine VviPrx31 peroxidase as a candidate gene involved in anthocyanin degradation in ripening berries under high temperature. J. Plant Res. 129, 513-526. doi: 10.1007/s10265-016-0786-3

Nakatsuka, T., Yamada, E., Saito, M., Fujita, K., and Nishihara, M. (2013). Heterologous expression of gentian MYB1R transcription factors suppresses anthocyanin pigmentation in tobacco flowers. Plant Cell Rep. 32, 1925-1937. doi: 10.1007/s00299-013-1504-4

Navrot, N., Rouhier, N., Gelhaye, E., and Jacquot, J. (2007). Reactive oxygen species generation and antioxidant systems in plant mitochondria. Physiol. Plant. 129, 185-195. doi: 10.1111/j.1399-3054.2006.00777.x

Pearse, I. S., Heath, K. D., and Cheeseman, J. M. (2005). Biochemical and ecological characterization of two peroxidase isoenzymes from the mangrove, Rhizophora mangle. Plant Cell Environ. 28, 612-622. doi: 10.1111/j.1365-3040.2005.01307.x

Requesens, D. V., Malone, R. P., and Dix, P. J. (2014). Expression of a barley peroxidase in transgenic apple (Malus domestica L.) results in altered growth, xylem formation and tolerance to heat stress. J. Plant Sci. 9, 58-66. doi: 10.3923/ jps.2014.58.66

Sedlák, E., Fabian, M., Robinson, N. C., and Musatov, A. (2010). Ferricytochrome c protects mitochondrial cytochrome $c$ oxidase against hydrogen peroxideinduced oxidative damage. Free Radic. Biol. Med. 49, 1574-1581. doi: 10.1016/j. freeradbiomed.2010.08.019

Shafi, A., Gill, T., Sreenivasulu, Y., Kumar, S., Ahuja, P. S., and Singh, A. K. (2015). Improved callus induction, shoot regeneration, and salt stress tolerance in Arabidopsis overexpressing superoxide dismutase from Potentilla atrosanguinea. Protoplasma 252, 41-51. doi: 10.1007/s00709-014-0653-9
Shaked-Sachray, L., Weiss, D., Reuveni, M., Nissim-Levi, A., and Oren-Shamir, M. (2002). Increased anthocyanin accumulation in aster flowers at elevated temperatures due to magnesium treatment. Physiol. Plant. 114, 559-565. doi: 10.1034/j.1399-3054.2002.1140408.x

Sinilal, B., Ovadia, R., Carmeli-weissberg, A. P. M., and Oren-shamir, M. (2011). Increased accumulation and decreased catabolism of anthocyanins in red grape cell suspension culture following magnesium treatment. Planta 234, 61-71. doi: 10.1007/s00425-011-1377-0

Štajner, D., and Popović, B. M. (2009). Comparative study of antioxidant capacity in organs of different Allium species. Cent. Eur. J. Biol. 4, 224-228. doi: 10.2478/ s11535-009-0010-8

Steyn, W. J., Holcroft, D. M., and Wand, S. J. E. (2004). Anthocyanin degradation in detached pome fruit with reference to preharvest red color loss and pigmentation patterns of blushed and fully red pears. J. Am. Soc. Hort. Sci. 129, $13-19$.

Steyn, W. J., Wand, S. J. E., Holcroft, D. M., and Jacobs, G. (2002). Anthocyanins in vegetative tissues: a proposed unified function in photoprotection. New Phytol 155, 349-361. doi: 10.1046/j.1469-8137.2002.00482.x

Thewes, F. R., Brackmann, A., Anese, R. D. O., Bronzatto, E. S., Schultz, E. E., and Wagner, R. (2017). Dynamic controlled atmosphere storage suppresses metabolism and enhances volatile concentrations of 'Galaxy' apple harvested at three maturity stages. Postharvest Biol. Technol. 127, 1-13. doi: 10.1016/j. postharvbio.2017.01.002

Ubi, B. E., Honda, C., Bessho, H., Kondo, S., Wada, M., Kobayashi, S., et al. (2006). Expression analysis of anthocyanin biosynthetic genes in apple skin: Effect of UV-B and temperature. Plant Sci. 170, 571-578. doi: 10.1016/j.plantsci.2005. 10.009

Vaknin, H., Bar-Akiva, A., Ovadia, R., Nissim-Levi, A., Forer, I., Weiss, D., et al. (2005). Active anthocyanin degradation in Brunfelsia calycina (yesterday-todaytomorrow) flowers. Planta 222, 19-26. doi: 10.1007/s00425-005-1509-5

Venter, M., Groenewald, J. H., and Botha, F. C. (2006). Sequence analysis and transcriptional profiling of two vacuolar H+-pyrophosphatase isoforms in Vitis vinifera. J. Plant Res. 119, 469-478. doi: 10.1007/s10265-006-0009-4

Xue, H., Zhang, F., Zhang, Z., Fu, J., Wang, F., Zhang, B., et al. (2015). Differences in salt tolerance between diploid and autotetraploid apple seedlings exposed to salt stress. Sci. Hortic. 190, 24-30. doi: 10.1016/j.scienta.2015.04.009

Zhang, J., Chen, C., Zhang, D., Li, H., Li, P., and Ma, F. (2014). Reactive oxygen species produced via plasma membrane NADPH oxidase regulate anthocyanin synthesis in apple peel. Planta 240, 1023-1035. doi: 10.1007/s00425-014-2120-4

Zhang, Z., Pang, X., Xuewu, D., Ji, Z., and Jiang, Y. (2005). Role of peroxidase in anthocyanin degradation in litchi fruit pericarp. Food Chem. 90, 47-52. doi: 10.1016/j.foodchem.2004.03.023

Zipor, G., Duarte, P., Carqueijeiro, I., Shahar, L., Ovadia, R., Teper-Bamnolker, P., et al. (2015). In planta anthocyanin degradation by a vacuolar class III peroxidase in Brunfelsia calycina flowers. New Phytol. 205, 653-665. doi: $10.1111 / \mathrm{nph} .13038$

Conflict of Interest Statement: The authors declare that the research was conducted in the absence of any commercial or financial relationships that could be construed as a potential conflict of interest.

Copyright (c) 2017 Rehman, You, Zhang, Goudia, Khan, Li and Ma. This is an open-access article distributed under the terms of the Creative Commons Attribution License (CC BY). The use, distribution or reproduction in other forums is permitted, provided the original author(s) or licensor are credited and that the original publication in this journal is cited, in accordance with accepted academic practice. No use, distribution or reproduction is permitted which does not comply with these terms. 